\title{
Multifaceted Roles of the Ras Guanine-Nucleotide Exchange Factor ChRgf in Development, Pathogenesis, and Stress Responses of Colletotrichum higginsianum
}

\author{
Qiongnan Gu, Meijuan Chen, Junbin Huang, Yangdou Wei, Tom Hsiang, and Lu Zheng
}

\begin{abstract}
First, second, and sixth authors: The Key Lab of Plant Pathology of Hubei Province, Huazhong Agricultural University, Wuhan 430070, Hubei, China; first and fourth authors: Department of Biology, University of Saskatchewan, Saskatoon S7N 5E2, Canada; and fifth author: School of Environmental Sciences, University of Guelph, Guelph N1G 2W1, Canada.
\end{abstract}

Current address of L. Zheng: The Key Lab of Plant Pathology of Hubei Province, Huazhong Agricultural University, Wuhan 430070, Hubei, China.

Accepted for publication 22 December 2016.

\begin{abstract}
The infection process of Colletotrichum higginsianum, which causes a disease of crucifers, involves several key steps: conidial germination, appressorial formation, appressorial penetration, and invasive growth in host tissues. In this study, the ChRgf gene encoding a Ras guanine-nucleotide exchange factor protein was identified by screening T-DNA insertion mutants generated from Agrobacterium tumefaciens-mediated transformation that were unable to cause disease on the host Arabidopsis thaliana. Targeted gene deletion of $C h R g f$ resulted in a null mutant $(\Delta C h r g f-42)$ with defects in vegetative growth, hyphal morphology, and conidiation, and poor surface

type and the mutant. The conidia of the mutant were unable to geminate on attached Arabidopsis leaves and did not cause any disease symptoms. Intracellular cyclic adenosine monophosphate levels in the $\Delta C h r g f$ mutant were lower than that of the wild type. Our results suggest that $C h R g f$ is a key regulator in response to salt and osmotic stresses in C. higginsianum, and indicate that it is involved in fungal pathogenicity. This gene seems to act as an important modulator upstream of several distinct signaling pathways that are involved in regulating vegetative growth, conidiation, infection-related structure development, and stress responses of $C$. higginsianum.
\end{abstract} attachment and low germination on hydrophobic surfaces; however, there were no apparent differences in appressorial turgor pressure between the wild
Additional keyword: morphogenesis.
The hemibiotrophic fungus Colletotrichum higginsianum is the causal agent of anthracnose disease of cruciferous plants, including many cultivated species of Brassica and Raphanus as well as the model plant Arabidopsis thaliana (Narusaka et al. 2004; O'Connell et al.2004). Once conidia of the pathogen attach to the plant surface, they germinate to produce germ tubes and quickly differentiate into melanized appressoria (O'Connell et al. 2012). The melanized appressoria accumulate significant turgor pressure that facilitates mechanical penetration of the host plant cell wall (O'Connell et al. 2012). Following penetration by $C$. higginsianum, biotrophic primary hyphae establish in first-invaded cells; however, these quickly differentiate into thin, tubular, necrotrophic secondary hyphae that kill and colonize neighboring cells (O'Connell et al. 2004). The C. higginsianum-A. thaliana pathosystem, in which both partners can be genetically manipulated and genome sequences are publicly available, provides a well-supported model system to examine hemibiotrophic fungus-plant interactions (O'Connell et al. 2004).

Surface recognition and attachment are two essential requirements for appressorial development. The mechanisms for recognition and adaptive response to environmental stimuli are well understood in several systems and appear to be similar in all eukaryotes (Kincaid 1991). Several signal transduction pathways such as cyclic adenosine monophosphate (cAMP) and mitogenactivated protein kinase (MAPK) have been shown to be involved in the regulation of appressorial formation and penetration in many

Corresponding author: L. Zheng; E-mail address: luzheng@mail.hzau.edu.cn

*The $\boldsymbol{e}$-Xtra logo stands for "electronic extra" and indicates that one supplementary table and four supplementary figures are published online.

(C) 2017 The American Phytopathological Society fungal species. cAMP-protein kinase A (PKA) signaling is important for initial surface recognition, appressorial formation, and turgor generation (Mehrabi et al. 2009; Mitchell and Dean 1995; Priyatno et al. 2012; Yang and Dickman 1999). In C. lagenarium, the RPK1 gene encoding the PKA regulatory subunit is involved in the formation of penetration hyphae. Mutations in genes involving adenylate cyclase or PKA have been shown to be defective in conidial germination and pathogenicity (Yamauchi et al. 2004). Involvement of a MAPK gene, $P M K 1$, in regulating late stages of appressorial development, penetration, and invasive growth has been well demonstrated in Magnaporthe oryzae (Thines et al. 2000). In C. lagenarium, a MAPK gene, $C M K 1$, is essential for conidial germination, appressorial formation, and invasive growth (Takano et al. 2000). Additionally, CoMEKKK1, a homolog of the yeast MAPK kinase STE11, is also involved in appressorial development in C. orbiculare (Sakaguchi et al. 2010). However, little is known about upstream regulators of cAMP and MAPK signaling pathways in $C$. higginsianum.

Ras proteins belong to the Ras superfamily of small GTPases which play critical roles in various cellular functions such as cell proliferation, cytoskeleton dynamics, membrane trafficking, and intracellular transport (Boguski and McCormick 1993). In previous studies, a Ras 2 protein was implicated in regulation of both the cAMP and the MAPK signaling pathways in M. oryzae (Zhou et al. 2014). In Fusarium graminearum, Ras2 is involved in hyphal growth and pathogenicity (Bluhm et al. 2007). In C. trifolii, Ct-Ras was shown to regulate conidial germination and hyphal growth (Ha et al. 2003). A C. trifolii strain expressing an active constitutive Ct-Ras failed to sporulate properly but produced appressoria during nutrient starvation (Memmott et al. 2002). A Rho family G protein, Ct-Rac, functions to restore the morphology of DARas by regulating the MAPK signaling pathway (Chen and Dickman 2004). Expression of a dominant negative $\mathrm{CtCdc} 42$ resulted in appressorial differentiation under 
noninductive conditions in $C$. trifolii. $\mathrm{CtCdc} 42$ likely functions as a downstream effector of Ct-Ras during spore germination and as a negative regulator of pathogenic development (Chen et al. 2006).

Ras proteins act as switches between on and off forms by binding to GTP or GDP, respectively. These are regulated by guanine exchange factors (GEF) and GTPase-activating proteins, respectively. Disruption of this balance may cause hypo- or hyperactivation of downstream signaling pathways (Boguski and McCormick 1993). In M. oryzae, a dominant allele, Ras ${ }^{\mathrm{G} 18 \mathrm{~V}}$ of Ras2, induces appressorial formation in aerial hyphae (Li et al. 2012). Sql2 is the only known RasGEF in Ustilago maydis, and it is involved in filamentous growth and virulence (Muller et al. 2003). In C orbiculare, the Ras GTPase-activating protein CoIra1 interacts with CoRas2 and is involved in appressorial formation and pathogenicity (Harata and Kubo 2014). To date, there has been no functional RasGEF gene identified in C. higginsianum.

The objective of this study was to identify and characterize a novel gene in $C$. higginsianum, $C h R g f$, encoding a RasGEF. We examined the expression of $C h R g f$ during appressorial formation, disrupted the gene function by T-DNA insertion and gene-replacement, and examined the effects on hyphal growth, conidiation, conidial surface attachment, and pathogenicity on A. thaliana.

\section{MATERIALS AND METHODS}

Fungal strains and culture conditions. $C$. higginsianum strain IMI349061 (Ch-1), which is fully virulent on A. thaliana Col-0 (Liu et al. 2013), was used as the reference wild-type strain in this study. This strain was originally isolated from diseased tissues of Brassica campestris and was obtained from CABI Bioscience (Egham, Surrey, UK). Ch-1 and all strains derived from it (Table 1) were maintained and cultured on potato dextrose agar (PDA) at $25^{\circ} \mathrm{C}$ under dark conditions. Five-day-old mycelia collected from potato dextrose broth (PDB) shaken at $180 \mathrm{rpm}$ was used for isolation of fungal DNA. Conidia washed from 7-day-old PDA cultures were used for transformation by the Agrobacterium tumefaciens-mediated transformation (AtMT) method (Liu et al. 2013). PDA was amended with hygromycin B (Roche, Mannheim, Germany) at $50 \mu \mathrm{g} / \mathrm{ml}$ or neomycin (Ameresco, Solon, $\mathrm{OH}$ ) at $150 \mu \mathrm{g} / \mathrm{ml}$ for selecting hygromycin-resistant or neomycinresistant transformants, respectively.

Molecular identification of $\boldsymbol{C h R g} \boldsymbol{f}$. In pathogenicity screening on Arabidopsis thaliana Col-0, a virulence-deficient mutant, G281, was obtained from an AtMT library of C. higginsianum wild-type strain Ch-1. Flanking sequences of the insertion site were isolated using the inverse polymerase chain reaction (PCR) method. The PCR products were cloned into the pMD18-T vector (TaKaRa, Dalian, China) and sequenced. The sequences were used to search against the $C$. higginsianum genome database (http://www.

TABLE 1. Wild-type and mutant strains of Colletotrichum higginsianum used in this study

\begin{tabular}{ll}
\hline Strain & \\
\hline WT & Wild-type strain of Colletotrichum higginsianum Ch-1 \\
G281 & AtMT transformant \\
$\Delta$ Chrgf-4 & ChRgf deletion mutant of Ch-1 \\
$\Delta$ Chrgf-14 & ChRgf deletion mutant of Ch-1 \\
$\Delta$ Chrgf-21 & ChRgf deletion mutant of Ch-1 \\
$\Delta$ Chrgf-39 & ChRgf deletion mutant of Ch-1 \\
$\Delta$ Chrgf-42 & ChRgf deletion mutant of Ch-1 \\
$\Delta$ Chrgf-46 & ChRgf deletion mutant of Ch-1 \\
$\Delta$ Chrgf-47 & ChRgf deletion mutant of Ch-1 \\
$\Delta$ Chrgf-49 & Ectopic strain \\
$\Delta$ Chrgf-54 & ChRgf deletion mutant of Ch-1 \\
$\Delta$ Chrgf-56 & ChRgf deletion mutant of Ch-1 \\
$\Delta$ Chrgf-58 & Ectopic strain \\
$\Delta$ Chrgf-59 & ChRgf deletion mutant of Ch-1 \\
$\Delta$ Chrgf-60 & ChRgf deletion mutant of Ch-1 \\
C $\Delta$ Chrgf-42 & ChRgf complementation strain from $\Delta$ Chrgf-42 \\
\hline
\end{tabular}

broadinstitute.org/annotation/genome/colletotrichum_group/ MultiHome.html) to obtain a full-length sequence of $C \bar{h} R g f$.

Deletion and complementation of $\operatorname{ChRgf}$. To replace $C h R g f$, fragments of approximately 1,000 bp of upstream and downstream flanking sequences of the gene were amplified with primers F1S/ F1A and F2S/F2A, respectively (Supplementary Table S1). The resulting upstream PCR product was digested with HindIII/SalI and downstream PCR product was digested with XbalI/KpnI, then ligated with an HPH cassette from pMD18T-HYG. F1-HYG-F2 was digested with HindIII and $K p n I$ and ligated with pCAMBIA3300III to form the gene-replacement construct pNeo3300IIIRasGEF-KO. The construct was transferred into Agrobacterium tumefaciens strain EHA105, then transformed into the wild-type strain Ch-1. After transforming the replacement vector into $\mathrm{Ch}$-1, hygromycin-resistant transformants were isolated and tested for resistance to neomycin, and subsequently screened by PCR with the primer pair F1S/F2A.

For complementation assays, the 4.6-kb PCR product, containing a 1.5 -kb upstream sequence, a full-length $C h R g f$ coding region, and a $0.94-\mathrm{kb}$ downstream sequence, was amplified from $\mathrm{Ch}-1$ genomic DNA using primers RgfS and RgfA and cloned into the pMD18-T vector (TaKaRa). The HindIII-digested PCR fragment was then ligated to the HindIII site in pCAMBIA3300III to obtain pNeo3300IIIRasGEF-C. The EHA105 strain with the complementation vector was transformed using AtMT by coculture with the $\Delta C h r g f$ strain. Transformants were selected on PDA supplemented with antibiotic G418 at $150 \mu \mathrm{g} / \mathrm{ml}$.

Phenotypic analysis. To analyze vegetative growth, mycelial plugs (5 $\mathrm{mm}$ in diameter) from 7-day-old PDA cultures were subcultured onto fresh PDA, then incubated at $25^{\circ} \mathrm{C}$ under dark conditions. The radial growth of vegetative mycelia was measured after 3 and 7 days of incubation. Osmotic and cell wall integrity assays were carried out on PDA agar plates supplemented with $0.7 \mathrm{M} \mathrm{NaCl}, 1.2 \mathrm{M} \mathrm{KCl}$, Calcofluor white (Sigma, Darmstadt, Germany) at $200 \mu \mathrm{g} / \mathrm{ml}$, Congo red (Yuanhang Reagent, Shanghai, China) at $200 \mu \mathrm{g} / \mathrm{ml}$, and $1 \mathrm{M}$ sorbitol (Sinopharm Chemical Reagent, Shanghai, China). Inhibition rates were calculated as follows: [(mean colony diameter on unamended media - mean colony diameter on compound-amended media)/mean colony diameter on unamended media].

For spore production, strains were grown on PDA at $25^{\circ} \mathrm{C}$ in dark conditions for 7 days. Conidia were harvested from three mycelial discs ( $9 \mathrm{~cm}$ in diameter) by suspending them in $5 \mathrm{ml}$ of sterile distilled water and centrifuging at 3,000 rpm for $2 \mathrm{~min}$. The spore suspensions were examined with a hemocytometer and adjusted to the target concentration of $1 \times 10^{5}$ conidia/ml with sterile distilled water.

For conidial germination and appressorial formation, $10 \mu \mathrm{l}$ of conidial suspension $\left(1 \times 10^{5} \mathrm{conidia} / \mathrm{ml}\right)$ was placed on plastic cover slips and incubated at $25^{\circ} \mathrm{C}$ for $24 \mathrm{~h}$. Conidial germination and appressorial formation were assessed by microscopic examination of at least 300 conidia or appressoria.

For assays of appressorial formation induced by chemicals, exogenous cAMP, 3-isobutyl-1-methylxanthine (IBMX), and 1,16hexadecanediol (Diol) were added to conidial suspensions (prepared as above) to final concentrations of $10 \mathrm{mM}, 2.5 \mathrm{mM}$, and $10 \mu \mathrm{M}$, respectively. Appressorial formation was assessed by microscopic examination of at least 300 appressoria.

For measuring appressorial turgor, a cytorrhysis assay described previously (Dixon et al. 1999; Howard et al. 1991) was used. Conidial suspensions of $10 \mu \mathrm{l}$ of $1 \times 10^{5}$ conidia/ml (prepared as above) were placed on plastic coverslips and incubated for $24 \mathrm{~h}$. After appressoria developed, the droplets of water were replaced with glycerol to a final concentration of 1 to $5 \mathrm{~mol} / \mathrm{liter}$. Following a 10-min incubation, the proportion of collapsed appressoria was assessed by microscopic examination of at least 300 appressoria.

Pathogenicity assay. The Arabidopsis thaliana ecotype Col-0 was used as a susceptible line in all pathogenicity assays. Arabidopsis plants were cultivated in growth chambers, as described by Liu 
et al. (2013). Four-week-old seedlings were used for plant infection assays.

For pathogenicity assays, $10 \mathrm{ml}$ of the spore suspension (prepared as above) was evenly sprayed onto attached Arabidopsis leaves. Inoculated plants were placed in a dark dew chamber at $25^{\circ} \mathrm{C}$ for $24 \mathrm{~h}$ with almost $100 \%$ relative humidity, then transferred to a $25^{\circ} \mathrm{C}$ growth chamber with a photoperiod of $12 \mathrm{~h}$. Lesion formation was examined after 3 to 5 days of incubation.

Plant infection assays were carried out using detached Arabidopsis leaves. The detached leaves were placed in plastic plates containing wet filter paper. Droplets of $10 \mu \mathrm{l}$ of conidial suspension (prepared as above) were placed on leaves. The plates were incubated in the dark at $25^{\circ} \mathrm{C}$ for 4 days. To observe the extent of fungal colonization, inoculated leaf tissues were cleared in a solution of methanol/chloroform/glacial acetic acid (6:3:1), followed by rehydration in water and staining with trypan blue (Bhadauria et al. 2010), and finally viewed by light microscopy.

Wounding experiments were carried out by lightly pricking detached leaves with a fine sterile needle prior to inoculation, and placing a conidial suspension $\left(10 \mu \mathrm{l}\right.$ of $5 \times 10^{4}$ conidia $\left./ \mathrm{ml}\right)$ directly on the wound sites. The leaves were incubated in the dark at $25^{\circ} \mathrm{C}$ for 4 days. Lesion formation was examined by light microscopy.

Quantification of intracellular cAMP. Intracellular cAMP levels were measured following Harata and Kubo (2014). Sevenday-old liquid mycelial cultures were harvested, frozen in liquid nitrogen, and lyophilized for $16 \mathrm{~h}$. The dried samples were ground to a fine powder in liquid nitrogen, resuspended in $200 \mathrm{ml}$ of ice-cold 6\% TCA (Trichloroacetic acid), and incubated on ice for $10 \mathrm{~min}$. After centrifugation at $4,000 \mathrm{rpm}$ for $15 \mathrm{~min}$ at $4^{\circ} \mathrm{C}$, the supernatant was collected and washed four times with five volumes of watersaturated ether. The remaining aqueous extract was lyophilized and dissolved in 6\% trichloroacetic acid. The cAMP levels were quantified following the manufacturer's instructions for the Monoclonal AnticAMP Antibody-Based Direct cAMP ELISA Kit (NewEast Biosciences, Malvern, PA).

DNA/RNA manipulation and quantitative reversetranscription PCR. Genomic DNA was extracted from vegetative hyphae using the cetyltrimethylammonium bromide protocol (Sambrook et al. 1989). General molecular biology techniques for nucleic acid analysis, including cloning, restriction digestion, gel electrophoresis, and ligation reaction, were performed following standard protocols (Sambrook et al. 1989). Southern blot analysis was carried out according to the protocol of the Amersham Gene Images Alkphos Direct Labeling and Detection System (GE Healthcare, Amersham, UK). The genomic DNA of Ch-1 was amplified with labeled primers Probe-s and Probe-a. Total RNA was extracted with the TaKaRa MiniBEST Universal RNA Extraction Kit (TaKaRa). Reverse-transcription assays were performed using the PrimeScript Double-Strand cDNA Synthesis Kit (TaKaRa).

For quantitative reverse-transcription (qRT)-PCR, total RNA of the mycelia was collected from hyphae grown for 4 days on PDA, or from infected Arabidopsis seedling tissues at different times $(0$, 22, 40, 66, and $90 \mathrm{~h}$ postinoculation [hpi]). Real-time RT-PCR mixtures were composed of $10 \mu \mathrm{l}$ of SYBR Green Realtime PCR Master Mix (Toyobo, Tokyo), $1 \mu \mathrm{l}$ of cDNA, 4-pmol concentration of each primer, and nuclease-free water to a final volume of $20 \mu \mathrm{l}$. The $\beta$-tubulin gene (CH063_01222.1) was amplified with primers $\beta 1$ and $\beta 2$ and used as an internal control for qRT-PCR. Primer pairs were designed for $C$. higginsianum gene $C h R g f$ and used to detect the gene expression levels. All results were normalized by $\beta$-tubulin expression, and relative changes in gene expression level were calculated by the comparative cycle threshold method (Applied Biosystems, Foster City, CA). Amplification conditions were as follows: $2 \mathrm{~min}$ at $95^{\circ} \mathrm{C}$, then 40 cycles consisting of $20 \mathrm{~s}$ at $95^{\circ} \mathrm{C}$, $15 \mathrm{~s}$ at $56^{\circ} \mathrm{C}$, and $20 \mathrm{~s}$ at $72^{\circ} \mathrm{C}$. For each gene, data were taken from three independent biological replicates.

Sequence analyses. BLAST programs were used to search for ChRgf homologs of C. higginsianum at the National Center for
Biotechnology Information (NCBI) and the Broad Institute databases, and the highest matching sequences were downloaded. Protein sequence alignments of ChRgf and its downloaded sequences were performed using ClustalX 2.0.11 (Larkin et al. 2007). Phylogenetic analyses were conducted using Mega 4.0 (http://www.megasoftware.net/method) with the neighbor-joining algorithm. The percentage of replicate trees in which the associated sequences were clustered together in the bootstrap test $(1,000$ replicates) are shown on the branches. Protein domains were predicted using Pfam (http://pfam.xfam.org/) and protein domain structures were illustrated by DOG 2.0 (Ren et al. 2009).

Statistical analyses. All experiments for phenotypic analysis and pathogenicity assays were carried out with three replicates per treatment and repeated twice. In osmotic and cell-wall-integrity assays, arcsine square-root transformation of the inhibition rate data was used to normalize the data distribution for further statistical analyses. The data were subjected to analysis of variance and, when significant treatment differences were found $(P<0.05$ or 0.01$)$, means were compared by the test of least significant difference. In most cases where treatments did not differ between experiments and where there was no significant treatment-experiment interaction, the results from a representative sample are shown.

\section{RESULTS}

Identification of a pathogenicity gene, ChRgf, in C. higginsianum. For genome-wide screening and identification of genes involved in pathogenicity of $C$. higginsianum, more than 5,000 transformants were generated using the AtMT method and screened for the ability to cause disease on Arabidopsis tissues.
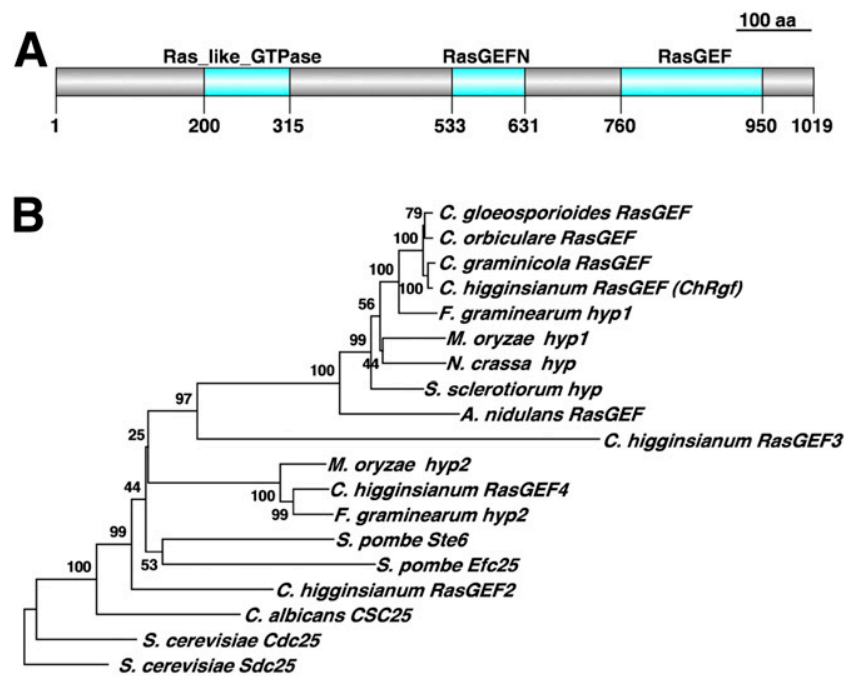

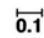

Fig. 1. Functional domain identification and phylogenetic tree. A, Prediction of domains in ChRgf. Light-shaded boxes depict the predicted Pfam domains of Ras_like_GTPase, RasGEF and RasGEFN in the protein. This figures was drawn using DOG 2.0. B, Phylogenetic tree of putative RasGEF identified in 11 fungal genomes. All of the RasGEF proteins were downloaded from the National Center for Biotechnology Information database and their accession numbers are as follows: Saccharomyces cerevisiae Cdc25, AAB67360.1 and Sdc25, CAA97461.1; Candida albicans Csc25, P43069.1; Schizosaccharomyces pombe Ste6, CAB40184.1 and Efc25, CAB58155.1; Fusarium graminearum Hyp1, XP_380827.1 and Hyp2, XP_391065.1; Aspergillus nidulans Hyp1, CBF89463.1; Neurospora crassa Hyp1, XP_957205.1; Colletotrichum gloeosporioides RasGEF, EQB45782.1; C. graminicola RasGEF, EFQ32199.1; C. higginsianum RasGEF1 (ChRgf), CCF47508.1, RasGEF2, CCF31834.1, RasGEF3, CCF35954.1, RasGEF4, and CCF38300.1; C. orbiculare RasGEF, ENH84347.1; Magnaporthe oryzae Hyp1, XP_003709741.1 and Hyp2, XP_003718916.1; and Sclerotinia sclerotiorum Hyp, XP_001588954.1. Numbers at branch nodes are bootstrap values out of 1,000 replications. 
Among transformants defective in virulence or pathogenicity, one mutant, G281, was identified as a nonpathogenic strain, defective in pathogenesis and occasionally forming flecks on detached and attached Arabidopsis leaves. No difference in disease severity was found between attached or detached leaves. To identify the location of the T-DNA insertion in the $C$. higginsianum genome, a $0.9-\mathrm{kb}$ genomic DNA flanking sequence was obtained using an inverse PCR strategy. The flanking sequence of the AtMT mutant G281 was used to search the $C$. higginsianum genome database (https://www. broadinstitute.org/annotation/genome/colletotrichum_group/ MultiHome.html) using BLASTx, pinpointing CH063_04179 (GenBank CCF47508.1) as the targeted sequence of the T-DNA insertion. The T-DNA insertion was located within the second exon of the CH063_04179 gene at 1,887 bp downstream of the predicted translational start site of the gene. Subsequently, the full length of the open reading frame was cloned from $\mathrm{Ch}-1$ (with introns). The predicted coding sequence was 3,276 bp in length and putatively encoded a 1,019-amino-acid protein with a predicted RasGEF domain (Fig. 1A); hence, the gene was designated ChRgf.

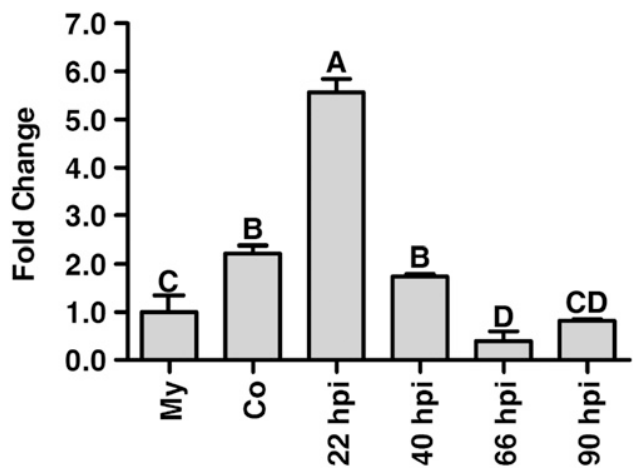

Fig. 2. Expression profiles of ChRgf at different infectious stages. RNA was extracted from fungal mycelia in vitro and from Arabidopsis seedlings at different times $(0,22,40,66$, and $90 \mathrm{~h}$ postinoculation [hpi]). Expression of a $\beta$-tubulin gene was used as an internal control. Relative abundance of ChRgf transcripts during various developmental stages postinoculation was normalized by comparing with the expression of ChRgf in fungal mycelia (relative transcript level $=1$ ) obtained from in vitro culture. Data were collected from three independent experiments with three replicates in each. The uppercase letter represents significant differences between in planta development stages and mycelia $(P<0.01)$. My $=$ mycelia and $\mathrm{Co}=$ conidia .
A BLASTp search for ChRgf homologs in the NCBI database revealed that the amino acid sequence of $C h R g f$ showed the highest similarities with RasGEF homologs present in other filamentous fungi, including C. graminicola (88\%), C. orbiculare (82\%), F. oxysporum (65\%), F. graminearum (64\%), M. oryzae $(57 \%)$, and Neurospora crassa (52\%). A phylogenetic tree of RasGEF-related proteins identified among various fungal genomes (Fig. 1B) indicated that ChRgf shares sequence homology with RasGEF of related fungi such as C. graminicola, C. gloeosporioides, and C. orbiculare, among others.

Upregulation of $\boldsymbol{C h R} \boldsymbol{R} f$ expression during appressorial formation. To gain insight into the functions of $C h R g f$, we first examined (by qRT-PCR) the gene expression profile at different developmental stages of $C$. higginsianum during pathogenesis. In comparison with relative expression levels of the vegetative-mycelial stage $(1.00 \pm 0.10$ compared with the control $\beta$-tubulin gene), expression of $C h R g f$ was significantly increased in germinating conidia ( 0 hpi, $2.22 \pm 0.16)$, and even more during appressorial formation (22 hpi, $5.56 \pm 0.28$ ) (Fig. 2).

Targeted deletion of the ChRgf in C. higginsianum. To determine whether the T-DNA insertion-mediated gene disruption of ChRgf might be responsible for the mutant G281 phenotype, targeted gene deletion of ChRgf was carried out using the genereplacement vector pNeo3300IIIRasGEF-KO that contains a hygromycin B phosphotransferase ( $h p h)$ gene cassette and 5' and 3' flanking sequences of ChRgf. pNeo3300IIIRasGEF-KO was introduced into the wild-type strain (Fig. 3A) and transformants were selected on medium containing hygromycin.

Among total 74 hygromycin-resistant transformants, 11 exhibited reduced growth rate compared with the wild-type strain. We selected these 11 transformants with reduced growth rate and two transformants showing wild-type growth for ChRgf gene detection by PCR. All 11 transformants with reduced growth rate lacked ChRgf amplifaction whereas, in the two transformants with wild-type growth, amplicons of the expected ChRgf size were detected (Fig. 3B).

Transformants $\Delta C h r g f-42$ and $\Delta C h r g f-59$ with reduced growth rate and the absence of the ChRgf amplicon were selected for Southern blot analysis of genomic DNA to further confirm gene deletion events. Southern blot analyses showed the absence of native $C h R g f$ in the genomes of the two selected trasnformants (Fig. $3 \mathrm{C})$. In Southern blotting, a band of approximately $4.3 \mathrm{~kb}$ was

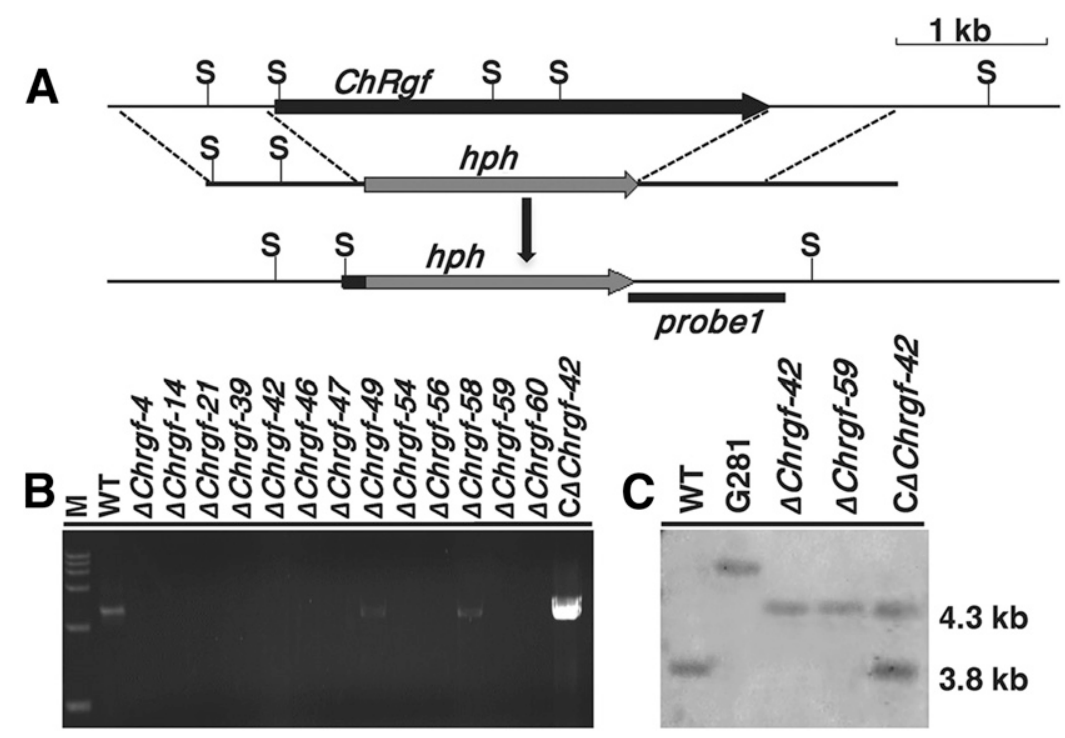

Fig. 3. Targeted gene deletion of ChRgf in Colletotrichum higginsianum. A, Strategic map of gene disruption vector construction and sites for restriction enzymes at the ChRgf genomic region. A bold line below the disruption construct represents the sequence used as a probe (probe 1) in Southern blot analysis. B, Polymerase chain reaction analysis of genomic DNA of the wild type (WT) and mutants. Lane M, DNA ladder DL5000. C, Southern hybridization analysis of the WT and mutants G281, $\Delta$ Chrgf-42, $\Delta$ Chrgf-59, and C $\Delta$ Chrgf-42. Genomic DNA was digested with SacI and separated in 0.8\% agarose gel. Blot was hybridized with the probe 1 amplified from genomic DNA of the WT. 
detected in the two transformants in contrast to a $3.8-\mathrm{kb}$ band in the wild-type strain (Fig. 3C), indicating that these two transformants ( $\Delta$ Chrgf-42 and $\Delta C h r g f-59$ ) had knockout mutations of the ChRgf. These two showed similar phenotypes, and $\Delta C h r g f-42$ was chosen for further biological characterization. The expression of $C h R g f$ was checked by qRT-PCR, and the results revealed that, in G281 and two knockout mutants, no indications of $C h R g f$ expression was detected; thus, we concluded that both the T-DNA insertional (G281) and two knockout ( $\Delta$ Chrgf-42 and $\Delta C h r g f-59)$ mutants had a null allele of the $C h R g f$.

A complementation assay was also carried out on the mutant $\Delta C h r g f-42$ with a full-length gene copy of $C h R g f$. Among resulting transformants confirmed by PCR exhibiting normal growth and conidiation comparable with the wild-type strain, we extracted genomic DNA of one complemented transformant, C $\Delta C h r g f-42$, for Southern blot analysis. Southern blotting revealed that the complemented strain $\mathrm{C} \Delta \mathrm{Chrgf}-42$ retained a copy of full-length ChRgf in addition to a disrupted $\Delta C h r g f$ sequence (Fig. 3C; Supplementary Fig. S2). The complemented strain C $\Delta C h r g f-42$ was similar in growth, conidiation, and virulence to that of the wild-type strain. Thus, strain $\mathrm{C} \Delta \mathrm{Chrgf-42}$ was used for further studies.

Effects of ChRgf disruption on vegetative growth, hyphal morphology, and conidiation. Growth and morphology of the wild-type strain and $C h R g f$ mutants were monitored on PDA plates for 7 days at $25^{\circ} \mathrm{C}$. Compared with the wild-type strain, the T-DNA insertion mutant G281 and the gene deletion mutant $\Delta$ Chrgf-42 formed smaller, uneven-edged colonies (Fig. 4A).
Examination by microscopy revealed that hyphal tips of mutants G281 and $\Delta$ Chrgf-42 were distorted with multiple branches, in contrast to more radial, linear hyphal growth of the wild type (Fig. 4B). Furthermore, hyphal growth was significantly inhibited in both mutants G281 and $\Delta C h r g f-42$, with growth rates approximately $60 \%$ of the wild type (Fig. 4C). The biomass from PDB was also measured. In agreement with results of colony growth on PDA plates, mutants G281 and $\Delta C h r g f-42$ displayed reduced growth in the liquid medium compared with the wild type (Fig. 4C).

Conidiation was examined in the wild type and the $C h R g f$ mutants on PDA after 7 days of incubation. Although there were no morphological defects observed in conidia of mutants G281 and $\Delta$ Chrgf-42 (Fig. 5A), conidial abundance was dramatically reduced in both mutants by 10 to $20 \%$ of the wild type (Fig. 4C). Normal growth was fully restored in the complementation strain $\mathrm{C} \Delta C h r g f-42$ (Fig. 4C).

Effects of $C h R g f$ disruption on appressorial formation and function. To examine infection-related morphogenesis, conidial germination and appressorial formation of $\Delta C h r g f-42$ were assessed during incubation on plastic hydrophobic coverslips. In the wild type and the complementation strain $\mathrm{C} \Delta \mathrm{Chrgf}-42$, more than $83 \%$ of conidia germinated and nearly all of them subsequently formed dark-brown appressoria after $24 \mathrm{~h}$ of incubation (Fig. 5A). However, in the mutants G281 and $\Delta C h r g f-42$, the conidia showed significantly lower germination rates (55 to 60\%) in comparison with the wild type and the complementation strain. In contrast,
A

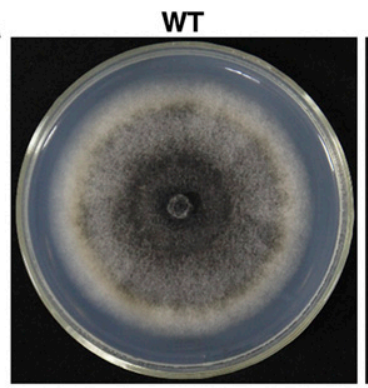

B

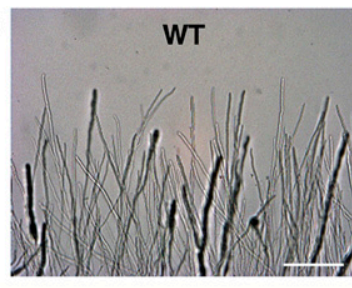

C

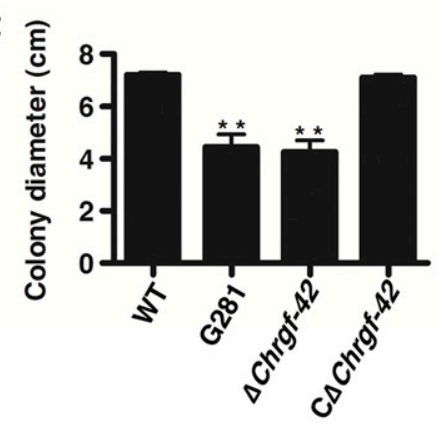

G281
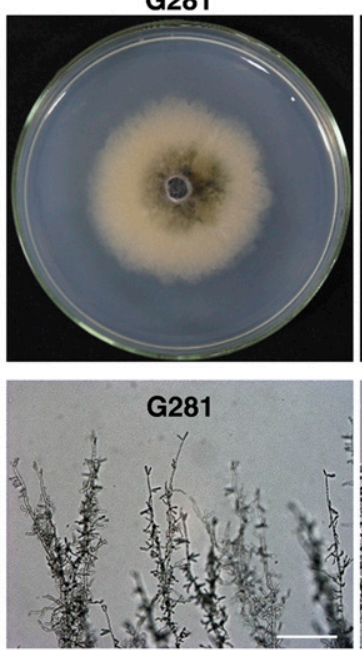

$\Delta$ Chrgf-42
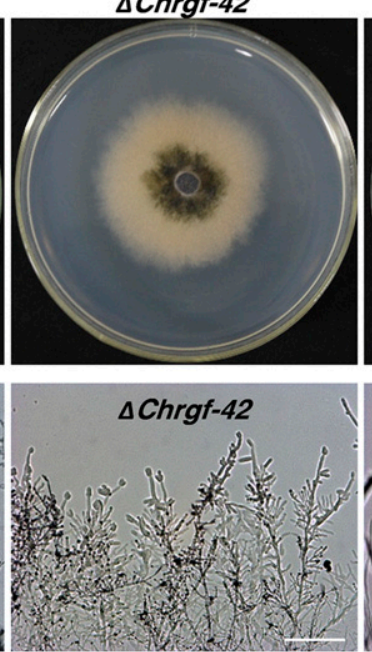
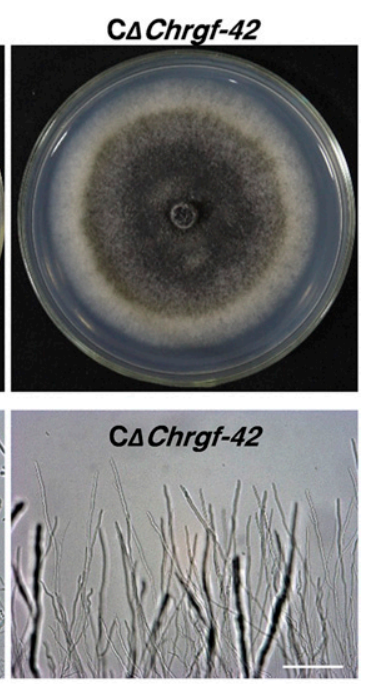
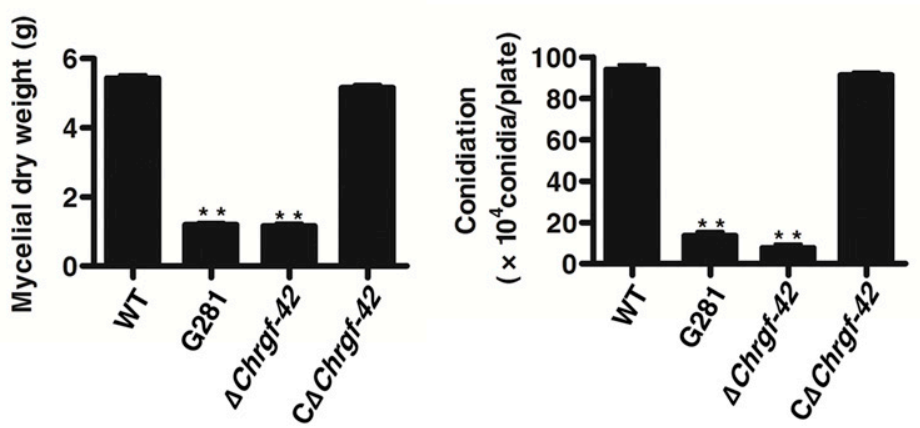

Fig. 4. Altered growth and sporulation of $\Delta C h r g f$ mutant. A, Colony morphology of the wild type (WT) and mutants G281, $\Delta C h r g f-42$, and C $\Delta C h r g f-42$ on potato dextrose agar (PDA) medium, photographed after 7 days of incubation in dark condition at $25^{\circ} \mathrm{C}$. The $\Delta C h r g f-42$ mutant displayed a lower mycelial growth rate and formed smaller, uneven-edged colonies compared with the WT and complemented strains. B, Hyphal tip growth and branching patterns of WT, G281, $\Delta C h r g f-42$, and C $\Delta$ Chrgf-42 on PDA plates after 4 days. Both mutants G281 and $\Delta C h r g f-42$ displayed a distorted hyphal morphology. Scale bar $=20 \mu \mathrm{m}$. C, Colony diameter, mycelial dry weight, and conidiation of WT, G281, $\Delta C h r g f-42$, and C $\Delta C h r g f-42$. Colony diameter (centimeters) was measured 7 days after incubation on PDA plates. Mean values were calculated from three replicates. Mycelia were collected from potato dextrose broth at 5 days postincubation and the dry weight of mycelia was measured. Conidiation was measured by collecting conidia grown from PDA plates at 7 days postincubation. Double asterisks represent significant difference between the mutant and WT $(P<0.01)$. 
fewer than $6 \%$ of the germinated conidia of G281 and $\Delta C h r g f-42$ mutants were able to differentiate into appressoria, and these were often associated with abnormally extended, long germ tubes (Fig. $5 \mathrm{~B})$. These results suggest important roles for $C h R g f$ in induction of conidial germination and appressorial formation.

Appressorial turgor pressure is required to penetrate the plant surface mechanically during infection. Thus, we further evaluated the appressorial turgor pressure in the wild type and the $\Delta C h r g f$ mutant using the glycerol cytorrhysis assay (Dixon et al. 1999; Howard et al. 1991). Results showed that proportions of the collapsed appressoria in each glycerol concentration in the $\Delta C h r g f$ mutant were similar to the wild type, indicating that $C h R g f$ was not involved in generation of the appressorial turgor pressure (Fig. 5C).

Role of $\boldsymbol{C h R g} \boldsymbol{f}$ in fungal pathogenicity. To assess the virulence of the $\Delta C h r g f$ mutant on A. thaliana, conidial suspensions of the wild type and mutants G281, $\Delta C h r g f-42$, and C $\Delta C h r g f-42$ were sprayed onto Col-0 plants. At 3 to 5 days postinoculation (dpi), the wild type or the complementation mutant $\mathrm{C} \Delta C h r g f-42$ caused disease symptoms on the Arabidopsis leaves, turning them yellow, then water soaked, and eventually the plants died after longer incubation. In contrast, mutants G281 and $\Delta$ Chrgf-42 lost the ability to cause extensive symptom development on inoculated plants, and only flecks occasionally appeared on the infected leaves (Fig. 6A).

Microscopic observation revealed that most conidia of G281 and $\Delta$ Chrgf-42 mutants were defective in germination (Fig. 6B and C), and only a few appressoria developed on inoculated leaf areas.
These appressoria rarely produced differentiated structures that penetrated into leaf epidermal cells by 5 dpi (Fig. 6B and C). In contrast, at 5 dpi with the wild-type and complementation strains, infected leaf epidermal cells were colonized with abundant primary and secondary invasive hyphae (Fig. 6B and C). These results indicated that the attenuated virulence of the mutants G281 and $\Delta C h r g f-42$ was due to a defect in conidial germination and appressorial formation.

To assess the ability of the mutants to grow invasively inside the host leaf tissues independent of the ability to penetrate, conidia of the wild type and mutants G281, $\Delta C h r g f-42$, and C $\Delta C h r g f-42$ were directly inoculated onto the wound sites of Arabidopsis leaves. On wounded inoculated tissues at $4 \mathrm{dpi}$, all strains produced extensive invasive hyphae within leaf epidermal cells, and both G281 and $\Delta$ Chrgf-42 caused leaf lesions with size comparable with that caused by the wild type and the complementation strain (Supplementary Fig. S1A). Microscopic analysis further revealed the presence of abundant invasive hyphae within epidermal cells of the wounded leaves infected by either G281 or $\Delta C h r g f-42$ (Supplementary Fig. S1B), suggesting that disruption of ChRgf did not affect invasive fungal growth within host tissues.

ChR $f$ acts upstream of the cAMP signaling pathway required for appressorial formation. cAMP regulates infection structure formation during fungal pathogenesis in some other fungi (Mehrabi et al. 2009; Mitchell and Dean 1995; Priyatno et al. 2012). Therefore, we examined the intracellular cAMP levels
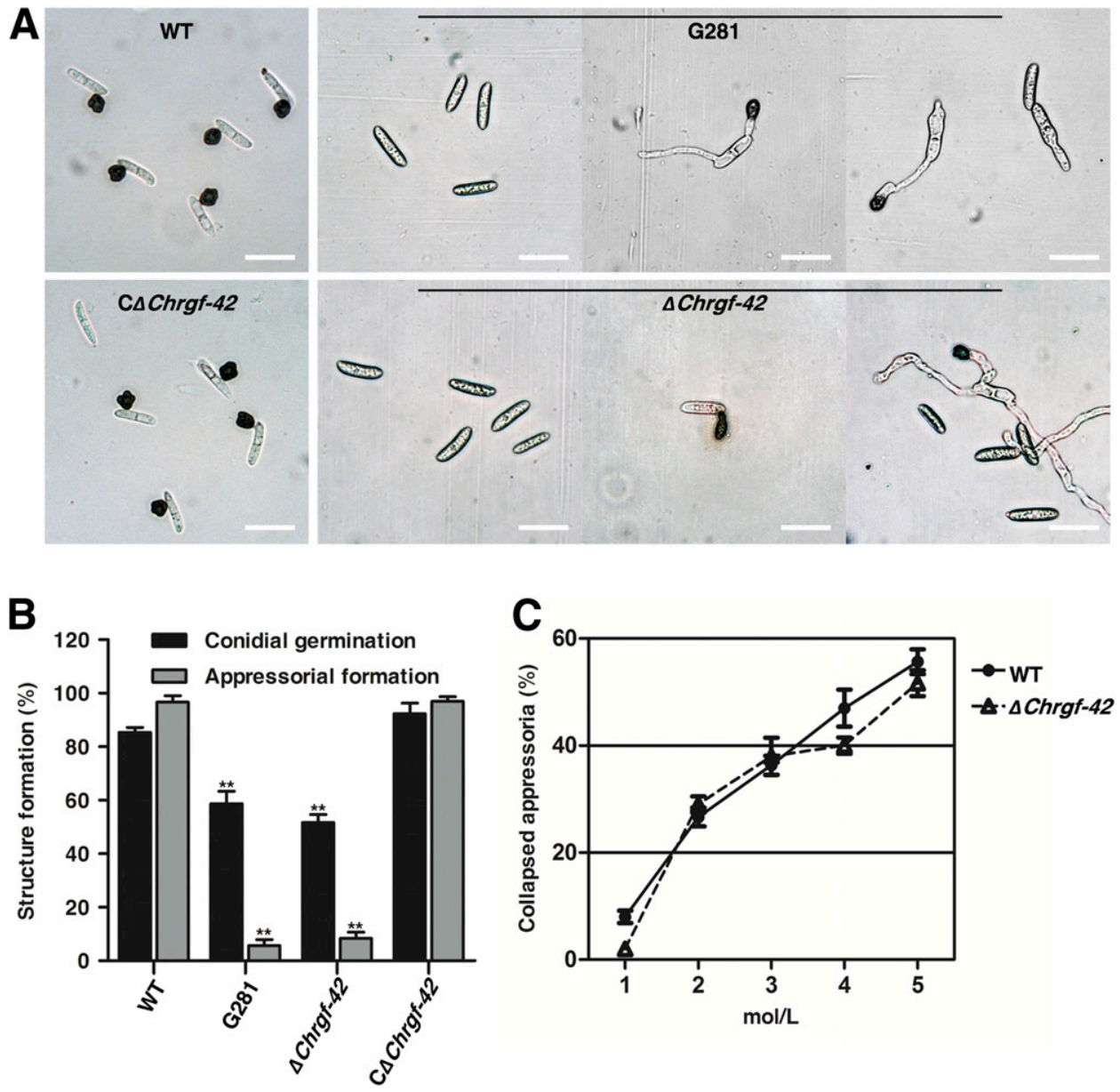

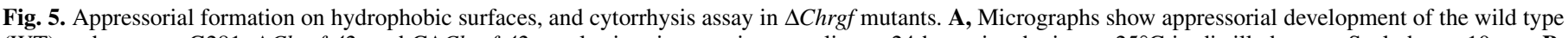

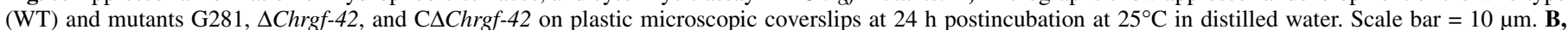

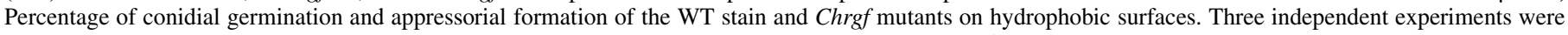

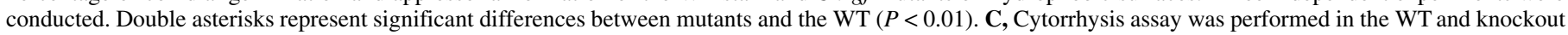

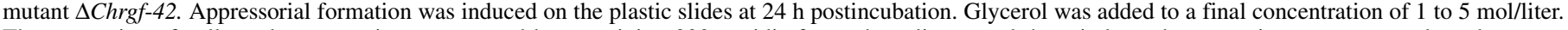
The proportion of collapsed appressoria was assessed by examining 300 conidia for each replicate, and three independent experiments were conducted. 
of the wild-type and ChRgf mutant strains in C. higginsianum. Compared with the wild-type and complementation strains, the intracellular cAMP levels were significantly reduced in the G281 and $\Delta C h r g f-42$ mutants, suggesting that $C h R g f$ may function upstream of the cAMP signaling pathway by regulating cAMP biosynthesis (Fig. 7A).

To test whether appressorial formation was responsive to exogenous chemical inducers, cAMP, IBMX, and Diol were added separately to the conidial suspensions that were incubated on hydrophobic coverslips for appressorial induction. IBMX is the most commonly used phosphodiesterase (PDE) inhibitor of cAMP and cyclic guanosine monophosphate (GMP) and, by inhibiting PDE, IBMX increases cellular cAMP and cGMP levels, thus significantly promoting appressorial formation (Kong et al. 2013). In nontreated controls, the formation of appressoria was significantly reduced in G281 and $\Delta C h r g f-42$ compared with the wild type and the complementation $\mathrm{C} \Delta C h r g f-42$ strain. In the presence of exogenous cAMP, approximately $80 \%$ of the conidia of $\Delta C h r g f$ germinated but formed abnormal germ tubes that did not further differentiate into normal appressoria; whereas, with additional cAMP, the wild-type strain continued to form normal appressoria with patterns similar to that of the untreated wild-type control. Under the same induction conditions, addition of IBMX was able to induce conidial germination in the $\Delta C h r g f-42$ mutant, and $70 \%$ of the germinated conidia differentiated into appressoria, although these appressoria were smaller and more elongated than the wild type. Similar results were observed in the treatment with Diol with the $\Delta C h r g f-42$ mutant. Diol induced conidia to geminate but they formed elongated germ tubes which failed to differentiate into appressoria (Fig. 7B). These results indicate that $C h R g f$ acted as a positive regulator in cAMP signaling during conidial germination and appressorial formation in $C$. higginsianum.

ChRgf regulates the interplay between the osmotic response and cell-wall-integrity signaling pathways. In addition to the impairments in conidial germination and appressorial formation, the $\Delta C h r g f-42$ mutants were also defective in mycelial growth and
A

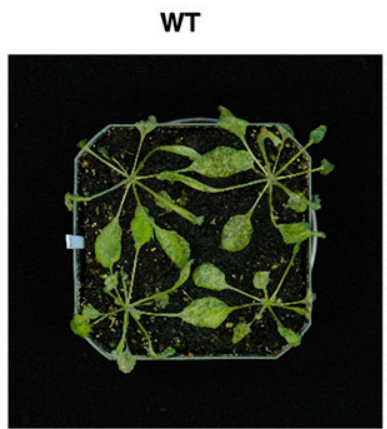

Day 3

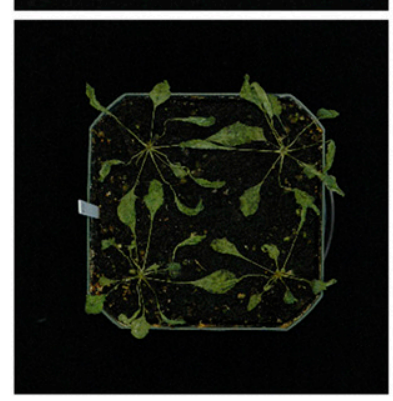

B
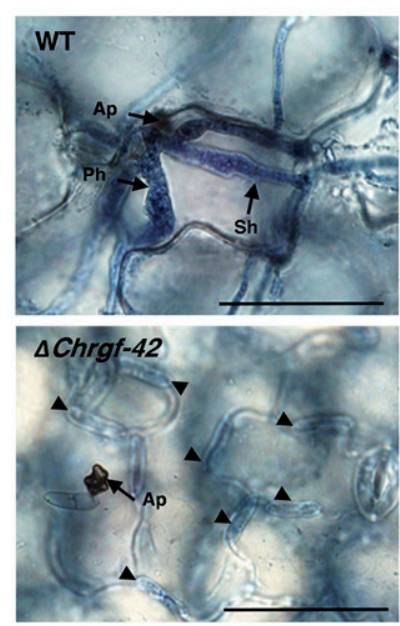

G281
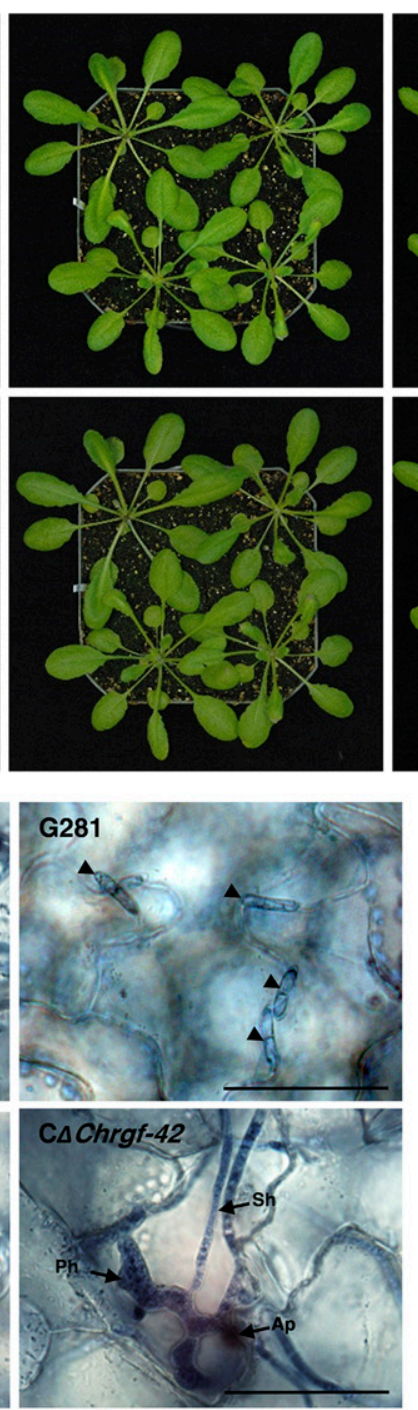

$\Delta$ Chrgf-42
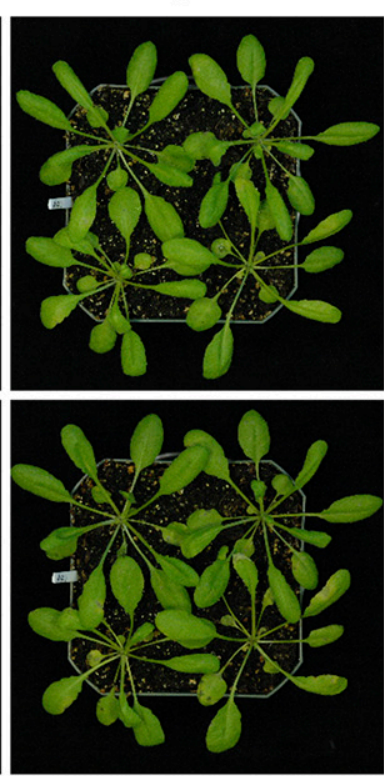

C
$\mathrm{C} \Delta$ Chrgf-42
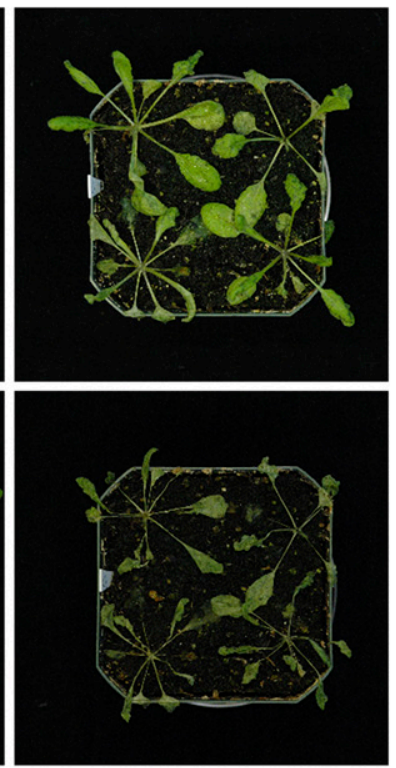

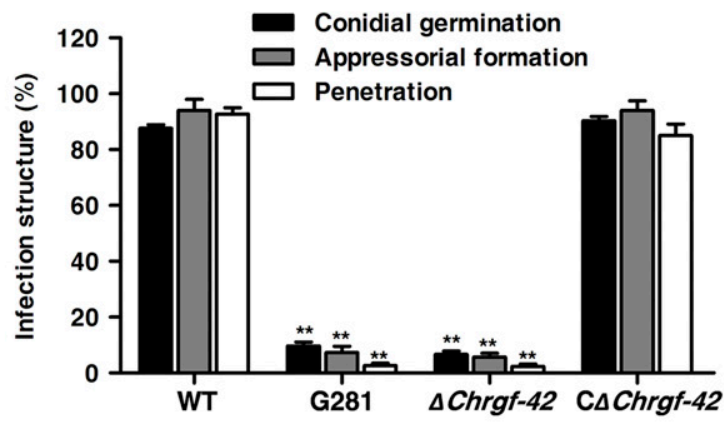

Fig. 6. Pathogenicity assay and observation of infection structure development in $\Delta$ Chrgf mutant on Arabidopsis. A, Arabidopsis plants were inoculated by spraying conidial suspensions of the wild type (WT) and mutants G281, $\Delta$ Chrgf-42, and C $\Delta$ Chrgf-42. Inoculated plants were photographed at 3 and 5 days postinoculation. B, Microscopic examination of fungal development on leaf tissues. Conidial suspensions of the WT and mutants G281, $\Delta$ Chrgf-42, and C $\Delta$ Chrgf42 were applied to the surface of intact plants and incubated at $25^{\circ} \mathrm{C}$. At 4 days postinoculation, inoculated leaves were fixed, stained with trypan blue, and photographed under a microscope. $\mathrm{Co}=$ conidia, $\mathrm{Ap}=$ appressoria, $\mathrm{Ph}=$ primary hyphae, and $\mathrm{Sh}=$ secondary hyphae. Scale bar $=40 \mu \mathrm{m} . \mathbf{C}$, Percentages of conidial germination, appressorial formation, and penetration rate of the WT and mutants G281, $\Delta$ Chrgf-42, and C $\Delta$ Chrgf-42 on Arabidopsis leaves. Approximately 100 conidia or appressoria were observed per incubated site. Three sets were examined in three independent experiments, and standard errors are shown. Double asterisks represent significant difference between mutants and the WT $(P<0.01)$. 
hyphal morphology. We further examined whether the $\Delta C h r g f-42$ mutant showed altered sensitivity upon treatment with various stressors. To test the integrity of the cell walls and the osmotic response of the $\Delta C h r g f-42$ mutant, mycelial growth was measured on PDA plates amended with various compounds that cause either cell wall or osmotic stresses, including $200 \mathrm{mM}$ Calcofluor white, $200 \mathrm{mM}$ Congo red, $1 \mathrm{M}$ sorbitol, $0.7 \mathrm{M} \mathrm{NaCl}$, and 1.2 M KCl. On media with Calcofluor white or Congo red, inhibition of mycelial growth was significantly higher in the $\Delta C h r g f-42$ mutant than that of the wild-type and complementation $\mathrm{C} \Delta C h r g f-42$ strains, suggesting that $C h R g f$ may function to maintain fungal cell wall integrity. Compared with the wild-type strain, the $\Delta C h r g f-42$ mutant showed less sensitivity to $\mathrm{NaCl}, \mathrm{KCl}$, or sorbitol (Fig. 8A), because growth inhibition of the $\Delta C h r g f-42$ mutant was lower than that of the wild type and the complementation strain $\mathrm{C} \Delta C h r g f-42$ (Fig. 8B). These results indicate that $C h R g f$ functions in the interplay between the cell wall response and the osmotic pathway.

\section{DISCUSSION}

Insertional mutagenesis is an effective way to identify novel genes involved in plant infection-related morphogenesis and

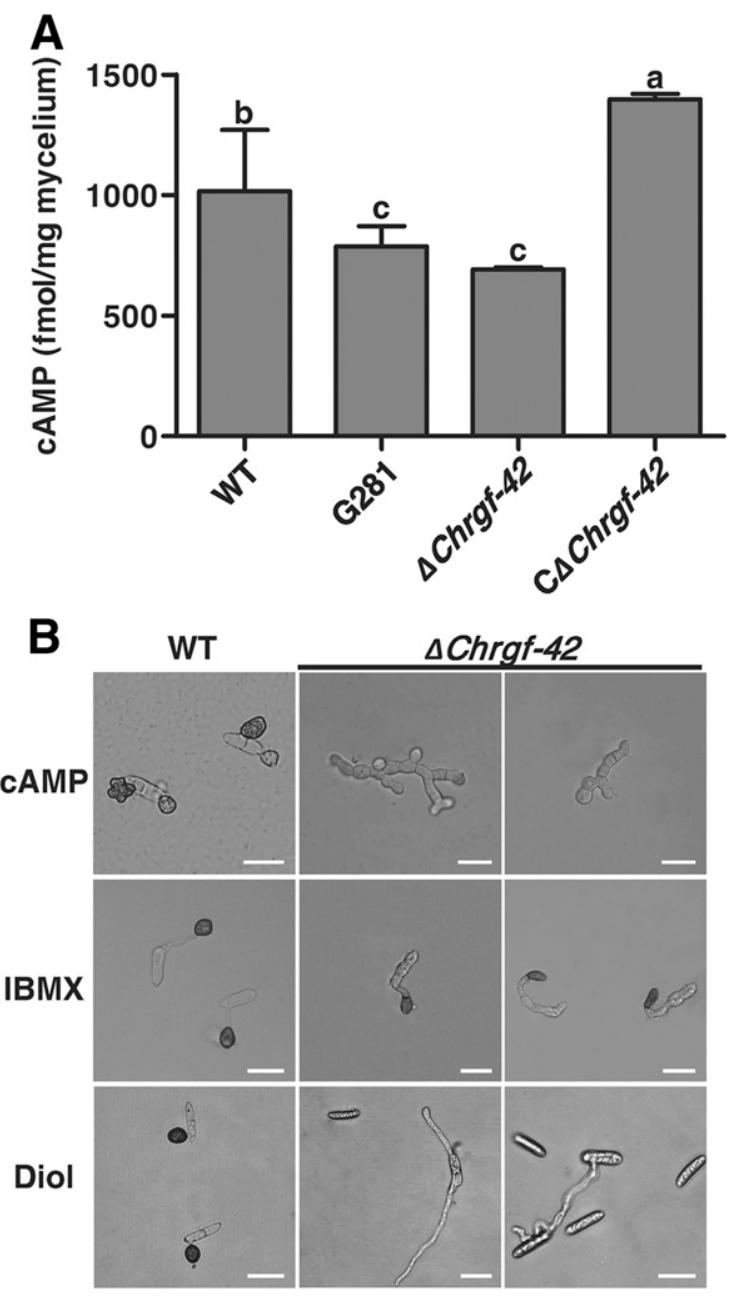

Fig. 7. ChRgf is functionally associated with the cyclic adenosine monophosphate (cAMP) signaling pathway. A, Measurement of intracellular cAMP levels. cAMP levels were measured in mycelia grown in liquid cultures of the wild type (WT) and mutants G281, $\Delta$ Chrgf-42, and C $\Delta$ Chrgf-42. Three independent experiments were conducted, and means with standard errors were calculated. B, Induction of appressorial formation of the WT and $\Delta C h r g f-42$ mutants by application of $10 \mathrm{mM}$ cAMP, $2.5 \mathrm{mM}$ 3-isobutyl-1-methylxanthine (IBMX), and $10 \mu \mathrm{M}$ 1,16-hexadecanediol (Diol), respectively. Scale bar = $10 \mu \mathrm{m}$. pathogenicity in fungal pathogens, including C. higginsianum (Huser et al. 2009). In this study, we identified a virulence gene, ChRgf of C. higginsianum, using Agrobacterium-meditated random insertional mutagenesis. We present several lines of evidence to demonstrate that $C h R g f$ played pleiotropic roles in regulating vegetative growth, conidiation, infection-related structure development, and stress responses of C. higginsianum (Fig. 9). Expression of $C h R g f$ also suggests that $C h R g f$ might be involved in early stages of $C$. higginsianum development during the infection process.

ChRgf encodes a structural homolog of Cdc25-like GEF in $C$. higginsianum. The deduced amino acid sequence contained a central C-terminal RasGEF domain and had high sequence similarity to other fungal RasGEF; in particular, Saccharomyces cerevisiae Sdc25 and Cdc25. In addition to a C-terminal RasGEF domain, the ChRgf has a second RasGEF domain at the N-terminal region (RasGEFN). In most cases, both RasGEF and RasGEFN domains catalyze guanine nucleotide exchange in Ras-like small GTPase proteins (Boguski and McCormick 1993). In different filamentous fungi, the functions of RasGEF are different. In budding yeast, only $C d c 25$ is essential for growth and activation of adenylyl cyclase. Sdc25 is dispensable for growth under normal culture conditions but the sdc25 mutant is defective in growth under nutrition-restricted conditions or when cultured on nonfermentable carbon sources (Boy-Marcotte et al. 1996; Damak et al. 1991). In U. maydis, the RasGEF proteins Sq12 is also essential for filamentous growth (Muller et al. 2003). In C. higginsianum, on the PDA media, the growth of the mutant was lower than the wild type, and the mycelial growth in liquid culture also had a lower yield. The observation was similar to those made for RasGEF proteins Cdc25 and Sdc25 in S. cerevisiae (Boy-Marcotte et al. 1996; Damak et al. 1991). Aside from the conserved catalytic domain, RasGEF in general are divergent, having other features such as $\mathrm{PH}$ domains and $\mathrm{SH} 3$ domains. Binding of the SH3 domain of RasGEF to proline-rich regions is important for signaling pathways that regulate cytoskeleton and Ras proteins (Leblanc et al. 1998; Mirey et al. 2005). A BLASTp search for ChRgf homologs in the Colletotrichum database revealed that there were three more RasGEF proteins in C. higginsianum. All of the four RasGEF had conserved RasGEF and RasGEFN domains (Supplementary Figs. S3 and S4) but the major differences were that only ChRas-GEF4 had a SH3 domain, and ChRgf had a special Ras-like_GTPase domain. The lack of grouping of ChRgf with known RasGEF in the dendrogram may have been due to the lack of the SH3 domain in ChRgf. We assume that this ChRgf is a RasGEF acting specifically on Ras proteins.

Our results showed that $C h R g f$ was required for disease development and multiple infection-related developmental processes, including vegetative growth, hyphal morphology, conidiation, conidial germination, appressorial formation, and pathogenicity. The lack of pathogenicity of G281 and $\Delta$ Chrgf-42 mutants was due to the reduced conidial germination and appressorial formation. This hypothesis is consistent with the observation that the G281 and $\Delta C h r g f-42$ mutants were unable to penetrate but able to cause invasive growth and disease lesions on wounded Arabidopsis leaves.

Germination of conidia is stimulated by surface hardness, hydrophobicity, and plant surface waxes and cutin (Kou and Naqvi 2016). On hard surfaces, germ tubes of the $C$. higginsianum wild type ceased elongation soon after emergence and developed appressoria. In contrast, $\Delta C h r g f-42$ germ tubes did not seem to be affected by the surface type, continued to elongate, and showed only a very low rate of appressorial formation. Furthermore, neither Arabidopsis leaves nor the cutin monomers increased the rate of appressorial formation in the $\Delta C h r g f-42$ mutants. Therefore, none of the physical and chemical surface signals were recognized by the mutants. In $M$. oryzae and $U$. maydis, signals required for appressorial formation include surface hydrophobicity and the presence of plant lipids (Mendoza-Mendoza et al. 2009; Tucker 

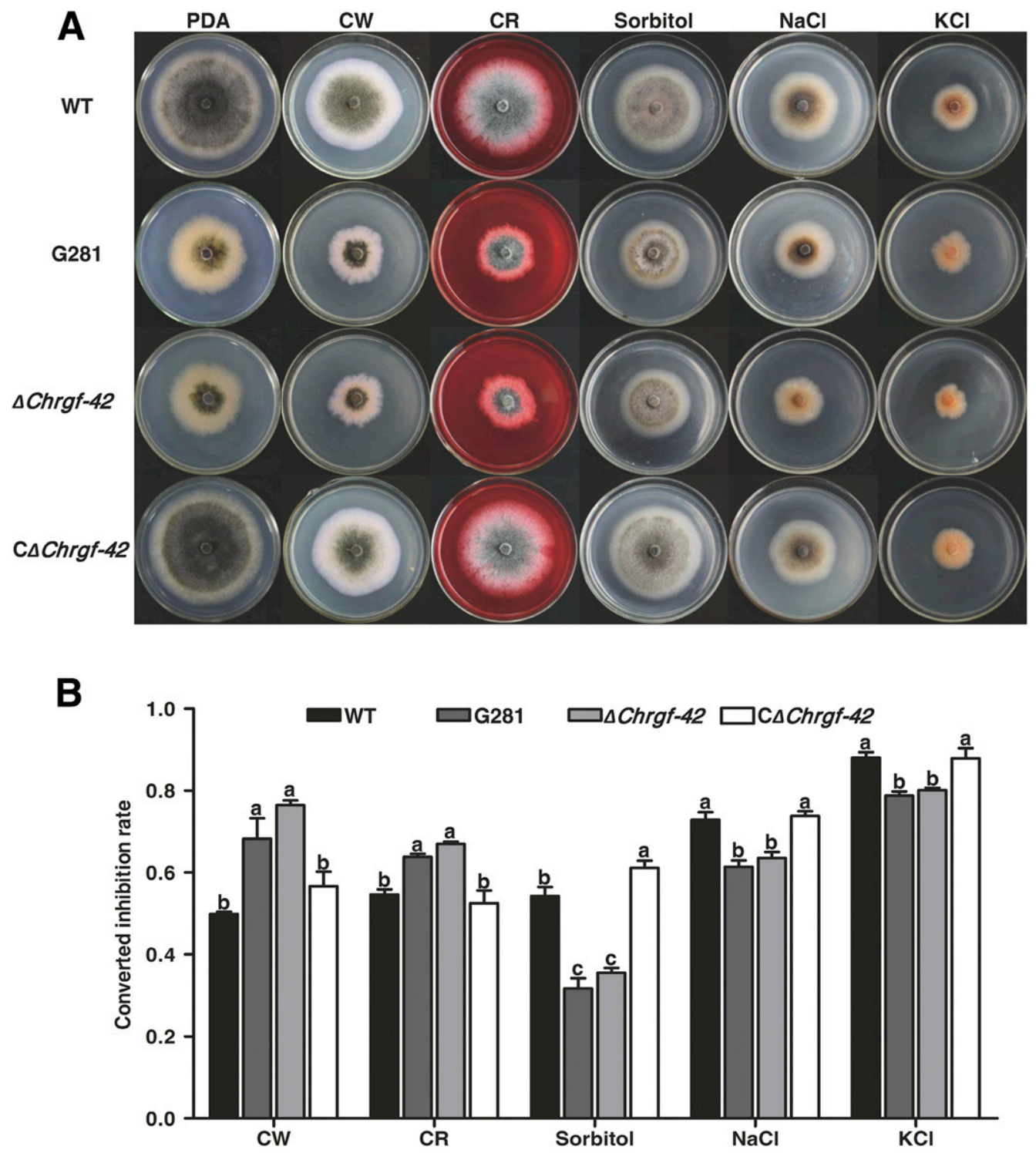

Fig. 8. Effects of cell wall damage and osmotic stresses on the $\Delta$ Chrgf mutant. A, The wild type (WT) and mutants G281, $\Delta C h r g f-42$, and C $\Delta C h r g f-42$ were incubated on potato dextrose agar plates supplemented with various stress inducers at $25^{\circ} \mathrm{C}$ for 7 days. Photographs show colony morphology of the strains grown on media supplemented with $0.7 \mathrm{M} \mathrm{NaCl}, 1.2 \mathrm{M} \mathrm{KCl}$, Calcofluor white (CW) at $200 \mu \mathrm{g} / \mathrm{ml}$, Congo red (CR) at $200 \mu \mathrm{g} / \mathrm{ml}$, or $1 \mathrm{M}$ sorbitol. B, Inhibition rates on colony growth by application of different stress inducers. Three independent experiments were conducted, data from original inhibition rates were arcsinesquare root transformed, and means with standard errors are shown. The lowercase letter represents significant difference between mutants and the WT $(P<0.05)$.

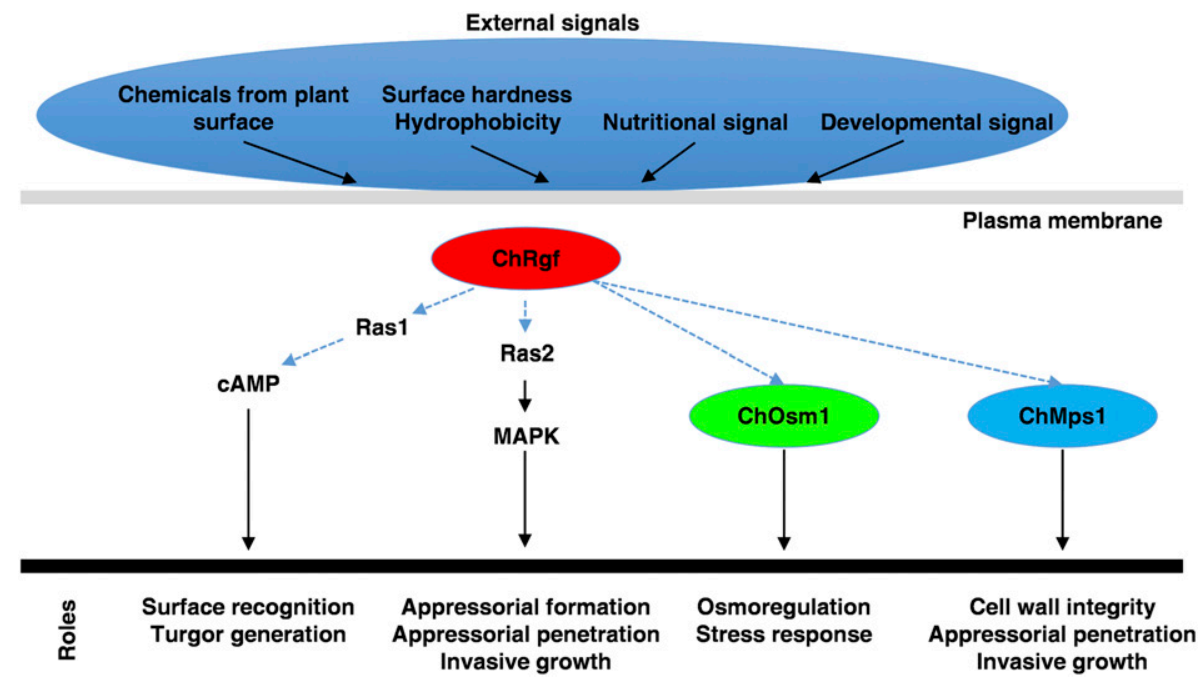

Fig. 9. Hypothetical model of the function of ChRgf in regulation of various signaling pathways. It is likely that $C h R g f$ acts as a key modulator upstream of several distinct signaling pathways that are involved in regulating infection-related structure development and stress responses of Colletotrichum higginsianum. 
and Talbot 2001). We postulate that $C h R g f$ mediates recognition or sensing of surface hardness in germ tubes for the initiation of penetration.

The defect in surface recognition also suggests that $C h R g f$ might be involved in cAMP signaling. The intracellular cAMP levels of the $\Delta C h r g f-42$ mutant were lower than that of the wild-type strain. Moreover, exogenous applications of cAMP and IBMX promoted the $\Delta C h r g f-42$ mutant to germinate or form appressoria on hydrophobic surfaces. Interestingly, our data showed that application of cAMP induced conidial germination but failed to effectively trigger appressorial formation on the hydrophobic surfaces. One explanation for this phenomenon could be that cAMP, a less lipophilic compound, is not easily absorbed into cells, given that the induction of appressorial formation requires high levels of intracellular cAMP. In contrast, IBMX application triggered appressorial formation in the $\Delta C h r g f$ mutant on the hydrophobic surface at $80 \%$ of the wild-type level. IBMX has been reported to be more efficient than cAMP in inducing appressorial formation in M. oryzae and acting as a specific inhibitor of PDE of stimulators of adenylate cyclase, and could induce a high endogenous level of cAMP accumulation (Mitchell and Dean 1995). Based on our experiments, we could not exclude the possibility of additional effects of IBMX on appressorial induction other than increasing the intracellular cAMP level.

Cdc25-like GEF are known to activate Ras proteins by catalyzing the GDP-GTP exchange in these proteins (Camonis et al. 1986). The role of Ras signaling in pseudohyphae growth and morphology was studied first in yeasts (Mösch et al. 1996) and then in several filamentous fungi, including Aspergillus nidulans, C. triffolii, U. maydis, and M. oryzae (Fillinger et al. 2002; Ha et al. 2003; Lee and Kronstad 2002; Muller et al. 2003; Zhou et al. 2014). Eukaryotic fungi usually have two Ras proteins, Ras1 and Ras2 (Bluhm et al. 2007; Mösch et al. 1996; Muller et al. 2003). In $S$. cerevisiae, two different Ras genes (Raslp and Ras $2 p$ ) serve overlapping as well as distinct functions. Both Raslp and Ras $2 p$ function upstream of the adenylyl cyclase, but only Ras $2 p$ acts on the STE20/STE11/STE7/KSS1-MAPK module to regulate pseudohyphal differentiation (Mösch et al. 1996). In Schizosaccharomyces pombe, Ras1 activates the pheromone-responsive MAPK cascade during conjugation, and also regulates cell morphology independently of the MAPK cascade (Papadaki et al. 2002). Recent studies revealed that these dual roles of Ras 1 are controlled mainly by the action of two different Cdc25-like GEF, Ste6 and Efc25. Although Ste6 couples Ras1 to the MAPK pathway, Efc25 links Ras1 to the Cdc42 pathway (Papadaki et al. 2002; Tratner et al. 1997). The gene expression profile of Ras1 and Ras 2 in $\Delta C h r g f-42$ and the wild type was assessed by qRT-PCR. In comparison with the wild type, expression of ChRasl was significantly increased and expression of ChRas 2 was decreased (data not shown), suggesting that $C h R g f$ may regulate the Ras signaling pathways which, in turn, influence the downstream signaling pathways affecting appressorial formation and appressorial penetration.

Because the hyphal morphology of $\Delta C h r g f$ was distorted compared with the wild type, we investigated the growth response upon different levels of cell wall integrity and osmotic stress. We found that the $\Delta C h r g f$ mutants were highly sensitive to cell-wall-integrity stress but displayed an increased resistance to salinity stresses. In well-characterized yeast stress signaling, the high-osmolarity glycerol (HOG) pathway is mainly involved in adaptation of fungal cells to the hyperosmotic stress, whereas the cell-wallintegrity (CWI) pathway is activated under conditions leading to cell-wall instability. The HOG pathway could play different coregulating roles within the CWI pathway (Rodríguez-Peña et al. 2010). Our results showed that, in C. higginsianum, ChRgfis a key component in response to multiple stresses via regulating different signaling pathways, including HOG pathway, which contributes to osmoregulation. The hypersensitivity to cell wall stresses may also indicate that $C h R g f$ is involved in regulating the CWI pathway. In
C. higginsianum, ChRgf may act as a negative modulator of the HOG pathway and a positive regulator of the CWI pathway.

In summary, our results suggest that $C h R g f$ of $C$. higginsianum is a key regulatory component playing multifaceted functions in vegetative growth, conidiation, appressorial development, virulence, and responses to stressors. (Fig. 9).

\section{ACKNOWLEDGMENTS}

We thank D. Jiang for providing the vectors. This work was supported by grants funded by the National Natural Science Foundation of China (number 31101399) and the Specialized Research Fund for the Doctoral Program of Higher Education of China (number 20110146120033).

\section{LITERATURE CITED}

Bhadauria, V., Miraz, P., Kennedy, R., Banniza, S., and Wei, Y. 2010. Dual trypan-aniline blue fluorescence staining methods for studying fungus-plant interactions. Biotech. Histochem. 85:99-105.

Bluhm, B. H., Zhao, X., Flaherty, J. E., Xu, J. R., and Dunkle, L. D. 2007. RAS2 regulates growth and pathogenesis in Fusarium graminearum. Mol. Plant-Microbe Interact. 20:627-636.

Boguski, M. S., and McCormick, F. 1993. Proteins regulating Ras and its relatives. Nature 366:643-654.

Boy-Marcotte, E., Ikonomi, P., and Jacquet, M. 1996. SDC25, a dispensable Ras guanine nucleotide exchange factor of Saccharomyces cerevisiae differs from CDC25 by its regulation. Mol. Biol. Cell 7:529-539.

Camonis, J. H., Kalekine, M., Gondre, B., and Garreau, H. 1986. Characterization, cloning and sequence analysis of the CDC25 gene which controls the cyclic AMP level of Saccharomyces cerevisiae. Characterization, cloning and sequence analysis of the CDC25 gene which controls the cyclic AMP level of Saccharomyces cerevisiae. EMBO J. 5:375-380.

Chen, C., and Dickman, M. B. 2004. Dominant active Rac and dominant negative Rac revert the dominant active Ras phenotype in Colletotrichum trifolii by distinct signalling pathways. Mol. Microbiol. 51:1493-1507.

Chen, C., Ha, Y. S., Min, J. Y., Memmott, S. D., and Dickman, M. B. 2006. $\mathrm{Cdc} 42$ is required for proper growth and development in the fungal pathogen Colletotrichum trifolii. Eukaryot. Cell 5:155-166.

Damak, F., Boy-Marcotte, E., Le-Roscouet, D., Guilbaud, R., and Jacquet, M. 1991. SDC25, a CDC25-like gene which contains a RAS-activating domain and is a dispensable gene of Saccharomyces cerevisiae. Mol. Cell. Biol. 11:202-212.

Dixon, K. P., Xu, J. R., Smirnoff, N., and Talbot, N. J. 1999. Independent signaling pathways regulate cellular turgor during hyperosmotic stress and appressorium-mediated plant infection by Magnaporthe grisea. Plant Cell 11:2045-2058.

Fillinger, S., Chaveroche, M. K., Shimizu, K., Keller, N., and d'Enfert, C. 2002. cAMP and ras signalling independently control spore germination in the filamentous fungus Aspergillus nidulans. Mol. Microbiol. 44:1001-1016.

Ha, Y. S., Memmott, S. D., and Dickman, M. B. 2003. Functional analysis of Ras in Colletotrichum trifolii. FEMS Microbiol. Lett. 226:315-321.

Harata, K., and Kubo, Y. 2014. Ras GTPase activating protein CoIra1 is involved in infection-related morphogenesis by regulating cAMP and MAPK signaling pathways through CoRas 2 in Colletotrichum orbiculare. PLoS One 9:e109045.

Howard, R. J., Ferrari, M. A., Roach, D. H., and Money, N. P. 1991. Penetration of hard substrates by a fungus employing enormous turgor pressures. Proc. Natl. Acad. Sci. USA 88:11281-11284.

Huser, A., Takahara, H., Schmalenbach, W., and O'Connell, R. 2009. Discovery of pathogenicity genes in the crucifer anthracnose fungus Colletotrichum higginsianum, using random insertional mutagenesis. Mol. Plant-Microbe Interact. 22:143-156.

Kincaid, R. 1991. Signaling mechanisms in microorganisms: Common themes in the evolution of signal transduction pathways. Adv. Second Messenger Phosphoprotein Res. 23:165-184.

Kong, L.-A., Li, G.-T., Liu, Y., Liu, M.-G., Zhang, S.-J., Yang, J., Zhou, X.-Y., Peng, Y.-L., and Xu, J.-R. 2013. Differences between appressoria formed by germ tubes and appressorium-like structures developed by hyphal tips in Magnaporthe oryzae. Fungal Genet. Biol. 56:33-41.

Kou, Y., and Naqvi, N. I. 2016. Surface sensing and signaling networks in plant pathogenic fungi. Semin. Cell Dev. Biol. 57:84-92.

Larkin, M. A., Blackshields, G., Brown, N., Chenna, R., McGettigan, P. A., McWilliam, H., Valentin, F., Wallace, I. M., Wilm, A., and Lopez, R. 2007. Clustal W and Clustal X version 2.0. Bioinformatics 23:2947-2948.

Leblanc, V., Tocque, B., and Delumeau, I. 1998. Ras-GAP controls Rhomediated cytoskeletal reorganization through its $\mathrm{SH} 3$ domain. Mol. Cell. Biol. 18:5567-5578. 
Lee, N., and Kronstad, J. W. 2002. Ras2 controls morphogenesis, pheromone response, and pathogenicity in the fungal pathogen Ustilago maydis. Eukaryot. Cell 1:954-966.

Li, G., Zhou, X., and Xu, J. R. 2012. Genetic control of infection-related development in Magnaporthe oryzae. Curr. Opin. Microbiol. 15:678-684.

Liu, L., Zhao, D., Zheng, L., Hsiang, T., Wei, Y., Fu, Y., and Huang, J. 2013. Identification of virulence genes in the crucifer anthracnose fungus Colletotrichum higginsianum by insertional mutagenesis. Microb. Pathog. 64:6-17.

Memmott, S. D., Ha, Y., and Dickman, M. B. 2002. Proline reverses the abnormal phenotypes of Colletotrichum trifolii associated with expression of endogenous constitutively active Ras. Appl. Environ. Microbiol. 68: 1647-1651.

Mendoza-Mendoza, A., Berndt, P., Djamei, A., Weise, C., Linne, U., Marahiel, M., Vraně̌, M., Kämper, J., and Kahmann, R. 2009. Physical-chemical plant-derived signals induce differentiation in Ustilago maydis. Mol. Microbiol. 71:895-911.

Mirey, G., Soulard, A., Orange, C., Friant, S., and Winsor, B. 2005. SH3 domain-containing proteins and the actin cytoskeleton in yeast. Biochem. Soc. Trans. 33:1247-1249.

Mitchell, T. K., and Dean, R. A. 1995. The cAMP-dependent protein kinase catalytic subunit is required for appressorium formation and pathogenesis by the rice blast pathogen Magnaporthe grisea. Plant Cell 7:1869-1878.

Mösch, H. U., Roberts, R. L., and Fink, G. R. 1996. Ras2 signals via the Cdc42/Ste20/mitogen-activated protein kinase module to induce filamentous growth in Saccharomyces cerevisiae. Proc. Natl. Acad. Sci. USA 93:5352-5356.

Muller, P., Katzenberger, J. D., Loubradou, G., and Kahmann, R. 2003. Guanyl nucleotide exchange factor Sq12 and Ras2 regulate filamentous growth in Ustilago maydis. Eukaryot. Cell 2:609-617.

Narusaka, Y., Narusaka, M., Park, P., Kubo, Y., Hirayama, T., Seki, M., Shiraishi, T., Ishida, J., Nakashima, M., Enju, A., Sakurai, T., Satou, M., Kobayashi, M., and Shinozaki, K. 2004. RCH1, a locus in Arabidopsis that confers resistance to the hemibiotrophic fungal pathogen Colletotrichum higginsianum. Mol. Plant-Microbe Interact. 17:749-762.

O’Connell, R., Herbert, C., Sreenivasaprasad, S., Khatib, M., Esquerre-Tugaye, M. T., and Dumas, B. 2004. A novel Arabidopsis-Colletotrichum pathosystem for the molecular dissection of plant-fungal interactions. Mol. Plant-Microbe Interact. 17:272-282.

O'Connell, R. J., Thon, M. R., Hacquard, S., Amyotte, S. G., Kleemann, J., Torres, M. F., Damm, U., Buiate, E. A., Epstein, L., Alkan, N., Altmüller, J., Alvarado-Balderrama, L., Bauser, C. A., Becker, C., Birren, B. W., Chen, Z., Choi, J., Crouch, J. A., Duvick, J. P., Farman, M. A., Gan, P., Heiman, D., Henrissat, B., Howard, R. J., Kabbage, M., Koch, C., Kracher, B., Kubo, Y., Law, A. D., Lebrun, M.-H., Lee, Y.-H., Miyara, I., Moore, N., Neumann, U., Nordström, K., Panaccione, D. G., Panstruga, R., Place, M., Proctor, R. H., Prusky, D., Rech, G., Reinhardt, R., Rollins, J. A., Rounsley, S., Schardl, C. L., Schwartz, D. C., Shenoy, N., Shirasu, K., Sikhakolli, U. R., Stüber, K., Sukno, S. A., Sweigard, J. A., Takano, Y., Takahara, H., Trail, F., van der Does, H. C., Voll, L. M., Will, I., Young, S., Zeng, Q., Zhang, J.,
Zhou, S., Dickman, M. B., Schulze-Lefert, P., Ver Loren van Themaat, E., Ma, L.-J., and Vaillancourt, L. J. 2012. Lifestyle transitions in plant pathogenic Colletotrichum fungi deciphered by genome and transcriptome analyses. Nat. Genet. 44:1060-1065.

Papadaki, P., Pizon, V., Onken, B., and Chang, E. C. 2002. Two ras pathways in fission yeast are differentially regulated by two ras guanine nucleotide exchange factors. Mol. Cell. Biol. 22:4598-4606.

Priyatno, T. P., Abu Bakar, F. D., Kamaruddin, N., Mahadi, N. M., and Abdul Murad, A. M. 2012. Inactivation of the catalytic subunit of cAMPdependent protein kinase A causes delayed appressorium formation and reduced pathogenicity of Colletotrichum gloeosporioides. Sci. World J. 2012: Article ID 545784. doi:10.1100/2012/545784

Ren, J., Wen, L., Gao, X., Jin, C., Xue, Y., and Yao, X. 2009. DOG 1.0: Illustrator of protein domain structures. Cell Res. 19:271-273.

Rodríguez-Peña, J. M., García, R., Nombela, C., and Arroyo, J. 2010. The high-osmolarity glycerol (HOG) and cell wall integrity (CWI) signalling pathways interplay: A yeast dialogue between MAPK routes. Yeast 27: 495-502.

Sakaguchi, A., Tsuji, G., and Kubo, Y. 2010. A yeast STE11 homologue CoMEKK1 is essential for pathogenesis-related morphogenesis in Colletotrichum orbiculare. Mol. Plant-Microbe Interact. 23:1563-1572.

Sambrook, J., Fritsch, E. F., and Maniatis, T. 1989. Molecular Cloning: A Laboratory Manual. Cold Spring Harbor Laboratory Press, Cold Spring Harbor, NY.

Takano, Y., Kikuchi, T., Kubo, Y., Hamer, J. E., Mise, K., and Furusawa, I. 2000. The Colletotrichum lagenarium MAP kinase gene CMK1 regulates diverse aspects of fungal pathogenesis. Mol. Plant-Microbe Interact. 13: 374-383.

Thines, E., Weber, R. W., and Talbot, N. J. 2000. MAP kinase and protein kinase A-dependent mobilization of triacylglycerol and glycogen during appressorium turgor generation by Magnaporthe grisea. Plant Cell 12: 1703-1718.

Tratner, I., Fourticq-Esqueöute, A., Tillit, J., and Baldacci, G. 1997. Cloning and characterization of the $S$. pombe gene efc $25+$, a new putative guanine nucleotide exchange factor. Gene 193:203-210.

Tucker, S. L., and Talbot, N. J. 2001. Surface attachment and pre-penetration stage development by plant pathogenic fungi. Annu. Rev. Phytopathol. 39: 385-417.

Yamauchi, J., Takayanagi, N., Komeda, K., Takano, Y., and Okuno, T. 2004. cAMP-PKA signaling regulates multiple steps of fungal infection cooperatively with Cmk1 MAP kinase in Colletotrichum lagenarium. Mol. Plant-Microbe Interact. 17:1355-1365.

Yang, Z., and Dickman, M. B. 1999. Colletotrichum trifolii mutants disrupted in the catalytic subunit of cAMP-dependent protein kinase are nonpathogenic. Mol. Plant-Microbe Interact. 12:430-439.

Zhou, X., Zhao, X., Xue, C., Dai, Y., and Xu, J. R. 2014. Bypassing both surface attachment and surface recognition requirements for appressorium formation by overactive ras signaling in Magnaporthe oryzae. Mol. PlantMicrobe Interact. 27:996-1004 